\title{
Environmental vibration influence on the blade inspection by an optical scanning device
}

\author{
Ruirui Song ${ }^{1, a}, \mathrm{He} \mathrm{Xie}^{1, \mathrm{~b}}$, Gang Zhang ${ }^{1, \mathrm{c}}$, Wenlong Li ${ }^{1,2, ~ *}$ \\ ${ }^{1}$ State Key Laboratory of Digital Manufacturing Equipment and Technology, \\ Huazhong University of Science \& Technology, Wuhan, P. R. China \\ ${ }^{2}$ Guangdong HUST Industrial Technology Research Institute, \\ Dongguan, P. R. China \\ a260834471@qq.com \\ bxiehe@mail.hust.edu.cn \\ czhanggang@mail.hust.edu.cn \\ *wlli@mail.hust.edu.cn
}

Keywords: environmental vibration; intrinsic frequency; blade inspection; optical scanning

\begin{abstract}
Blades are critical parts of aero-engine and nuclear turbine, and it is important to inspect blades by optical method accurately. In this paper, an optical scanning device is designed for inspecting blades. In general, the main external factor affecting the accuracy in the process is environmental vibration, the following parts studied the influence of environmental vibration on the device. First, the characteristics of the environmental vibration are analyzed to find out the frequency range affecting the inspection accuracy. Second, the intrinsic frequency of the device is obtained by modal analysis. Third, the response of the device under external vibration is obtained by simulation experiment. At last, experiments show that the inspection accuracy is $0.0277 \mathrm{~mm}$ when the environmental vibration frequency is equal to the intrinsic frequency, which can meet the inspection requirements very well.
\end{abstract}

\section{Introduction}

Blade inspection using optical method is important in the field of computer vision and automation manufacturing. Optical scanning device can inspect the blade in a fast and automatic way. At the same time, this method has high accuracy and stability [1]. Recently, Breuckmann company produced a new optical device, which was enough to inspect geometric deformation of a blade by adopting the techniques of structured light projector [2]. GE company used a more time-saving and cost-saving control method rather than the conventional CMM method, which reduced the inspection time and obtained more conclusive and easily transferrable measurement results obviously [3]. Heo et al. presented a computer-aided measurement plan using a CMM based on the CAD model, which could divide the blade surface into several measuring parts [4]. Li and $\mathrm{Ni}$ proposed an iterative method of non-rigid registration for 2D blade section profile reconstruction [5].

For these optical scanning devices, environmental vibration is an important aspect affecting the accuracy of the device. The evaluation of environmental vibration is to ensure that the device error is in the allowable range [6]. Duan and Hao combined the finite element method with the multi-body system simulation method to establish the body dynamics model, and the main loads were obtained through simulation [7]. Celebi and Schmid used the Thin Layer Method/Flexible Volume Method and the Boundary Element Method to establish a three dimensional dynamic response model, then the validity of the methods were demonstrated by comparison with results of analytical solution [8]. Zhao and $\mathrm{Xu}$ established the virtual prototype model based on the mechanical system dynamics analysis software ADAMS. And it proved that the damping of the system effectively restrain the vibration through the simulation of vibration source [9]. 
In this paper, an optical blade inspection device named iBlade Scan I for casting/forging blades is designed, which meets the required inspection accuracy very well. Vibration is one of the most important environmental factors affecting the precision of the device, so it is necessary to identify the environmental vibration in required range. This paper is organized as follows: Different vibration sources affecting the inspection accuracy are found and sensitive vibration frequency range for precision device is established in Section 2 according to the national standard. Following that, the software ADAMS are used to obtain finite element model, intrinsic frequency and vibration model of the device. Inputs and outputs are defined to finish the simulation experiment of external vibration source in Section 3. Finally, experiments and conclusions are given in Section 4 and Section 5, respectively.

\section{Characteristics of Environmental Vibration}

Environmental vibration is caused by the different vibration sources, and the test results need to be compared with the allowable error to evaluate the accuracy of the device under environmental vibration. When the error of the device is less than the permissible error, the device can be used in the current environment conditions. Factors related to the environmental vibration include the direction of vibration, the frequency of the vibration and the time of the vibration.

The source of the environmental vibration mainly includes:

(1) Vibration caused by human production activities.

Traffic equipment, staff walking, and using of the power machinery equipment. This kind of vibration has wide frequency band and large amplitude, which has an increasing effect on precision device.

(2) Vibration caused by natural activities.

Typhoons, storms and other climate change. This kind of vibration is random, and the vibration frequency is low.

The environmental vibration frequencies of the main sources are shown in Table 1 [10].

Table 1. The environmental vibration frequencies of the main sources

\begin{tabular}{|c|c|c|}
\hline Vibration sources & Vibration frequency $(\mathrm{Hz})$ & Distance of vibration sources $(\mathrm{m})$ \\
\hline \multirow{2}{*}{ Train } & 30 & $400($ normal speed $)$ \\
\cline { 2 - 3 } & $20 \sim 25$ & $10 \sim 30(20 \mathrm{~km} / \mathrm{h})$ \\
\hline \multirow{2}{*}{ Car } & $10 \sim 25$ & $20 \sim 38($ no load $)$ \\
\cline { 2 - 3 } & $5 \sim 15$ & $20 \sim 38($ full-load $)$ \\
\hline Staff walking & $1 \sim 3$ & nearby \\
\hline Power machinery equipment & $10 \sim 50$ & nearby \\
\hline Natural activities & $0.1 \sim 0.5$ & nearby \\
\hline
\end{tabular}

The measured environmental vibration shows that all kinds of vibration wave generated by vibration sources are random and superposition. According to existing environmental vibration standards at home and abroad, and considering the characteristics of different vibration sources, vibration sensitive frequency range for precision device is $1 \sim 150 \mathrm{~Hz}$.

\section{Modal analysis of device}

3.1 Finite element model of the device. The structure of the device is complex, so actual structure need to be simplified for convenience. The simplified model is shown in Fig. 1. The parts of the device can be divided into grid, and the finite element model is shown in Fig. 2. 


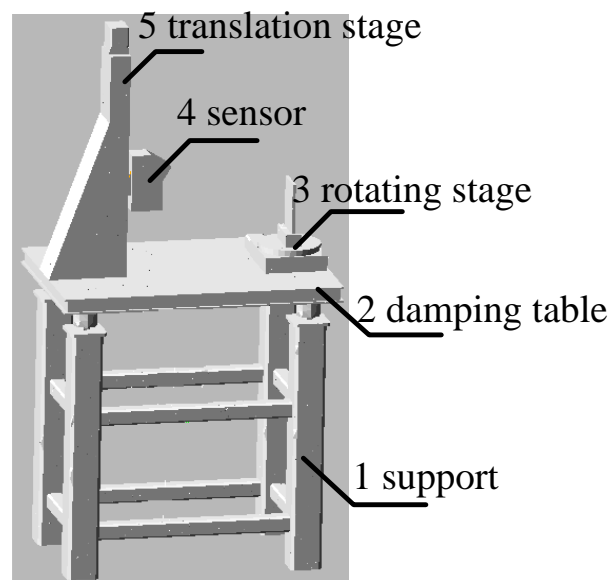

Figure 1. The simplified model of the device

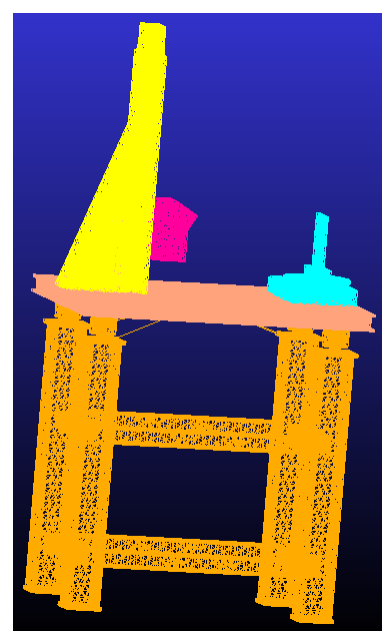

Figure 2. Finite element model of the device

According to the actual situation, the material properties (density, yonug's modulus, Poisson's ratio) of the device can be specified and they are shown in Table 2.

Table 2. The material properties of the device

\begin{tabular}{|c|c|c|c|c|}
\hline Number & 1 & 2 & 4 & 3,5 \\
\hline Material & Mild steel & Stainless steel & Plastic & Aluminum \\
\hline Density $\left(\mathrm{g} / \mathrm{cm}^{3}\right)$ & 7.84 & 7.93 & 1.05 & 2.69 \\
\hline Yonug's modulus $\left(\mathrm{kN} / \mathrm{mm}^{2}\right)$ & 200 & 193 & 2.2 & 69 \\
\hline Poisson's ratio & 0.26 & 0.247 & 0.394 & 0.33 \\
\hline
\end{tabular}

3.2 Modal analysis of device. Modal analysis is used to calculate the intrinsic frequency and vibration model of the device, and it is also the precondition of random vibration analysis. The former 6 modal vibration modes and intrinsic frequency can be obtained by Adams. The former 6 modal intrinsic frequency is shown in Table 3.

Table 3. The former 6 modal intrinsic frequency

\begin{tabular}{|c|c|c|c|c|c|c|}
\hline Order & 1 & 2 & 3 & 4 & 5 & 6 \\
\hline Frequency $(\mathrm{Hz})$ & 19.68 & 24.23 & 33.49 & 49.64 & 55.40 & 63.20 \\
\hline
\end{tabular}

The former 6 modal intrinsic frequency are all in the range of the vibration of the factory environment. The results of modal vibration modes are shown in Fig. 3.

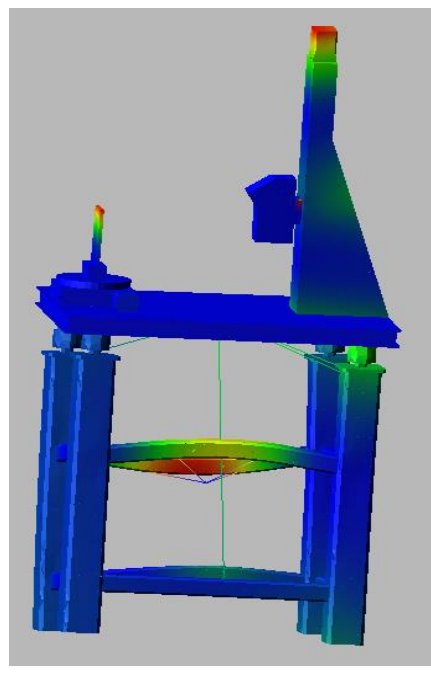

(a)

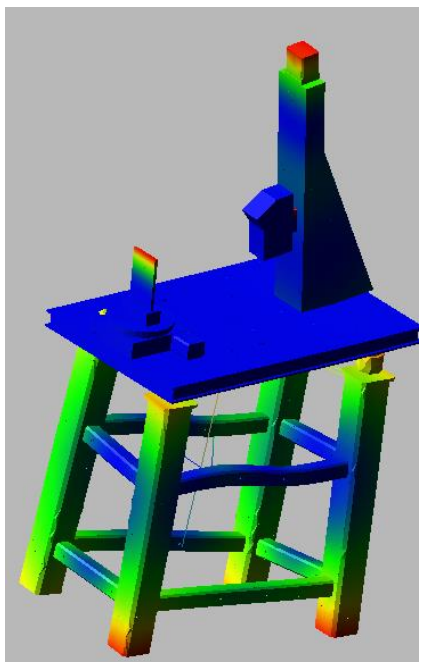

(b)

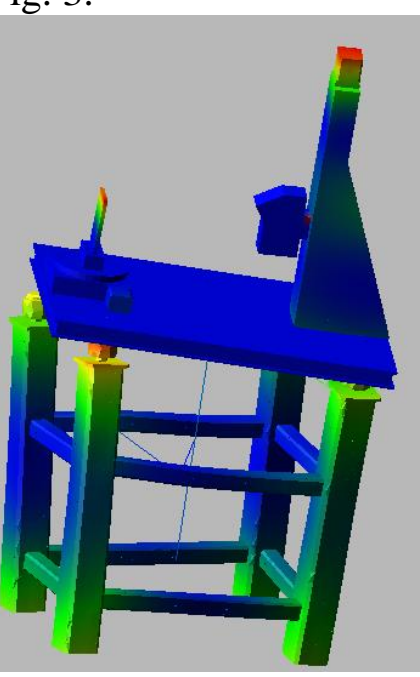

(c) 


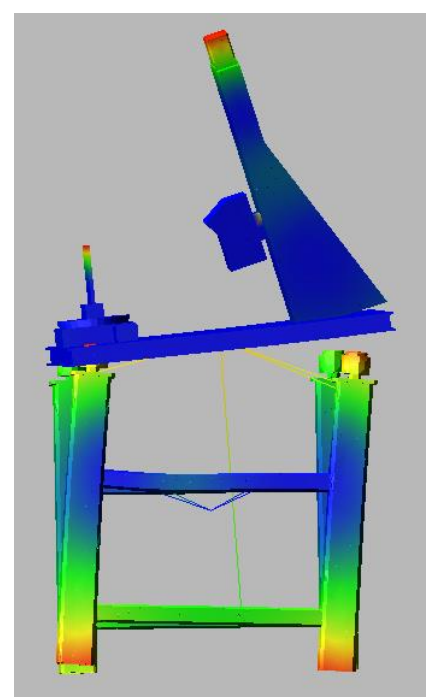

(d)

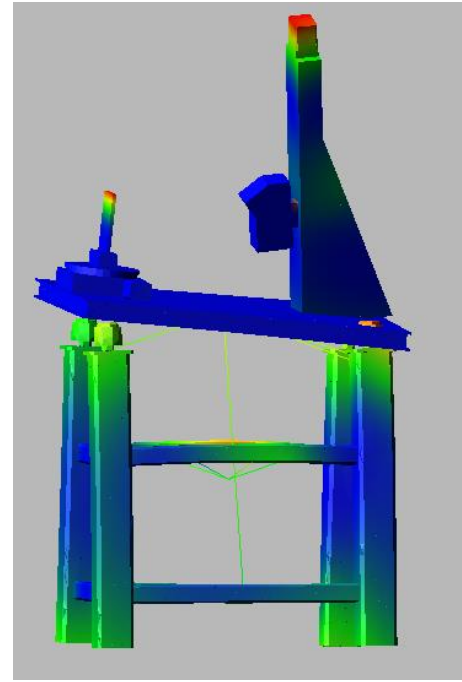

(e)

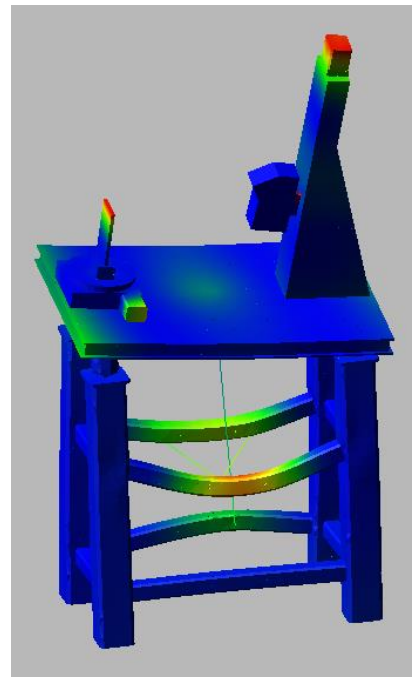

(f)

Figure 3. The former 6 vibration modes

The former 6 vibration modes show that the maximum vibration of the device is at the top of rotating stage and translation stage. It is necessary to test the effect of environment vibration on the device, especially at the top of rotating stage and translation stage.

3.3 Simulation of vibration analysis. For a more real simulation of environmental vibration, the vibration source will be put on the support of the device. In order to measure the whole influence of vibration more accurately, there are different measurement points from top to bottom on the rotating stage and translation stage. Inputs $1 \sim 2$ and outputs $3 \sim 8$ are defined in the device, respectively. Vibration of the device includes vertical and horizontal directions. Thus, there are two different vibration directions at input 1 and 2 . The vibration frequencies are all $0 \mathrm{~Hz} \sim 1000 \mathrm{~Hz}$ in following experiments.

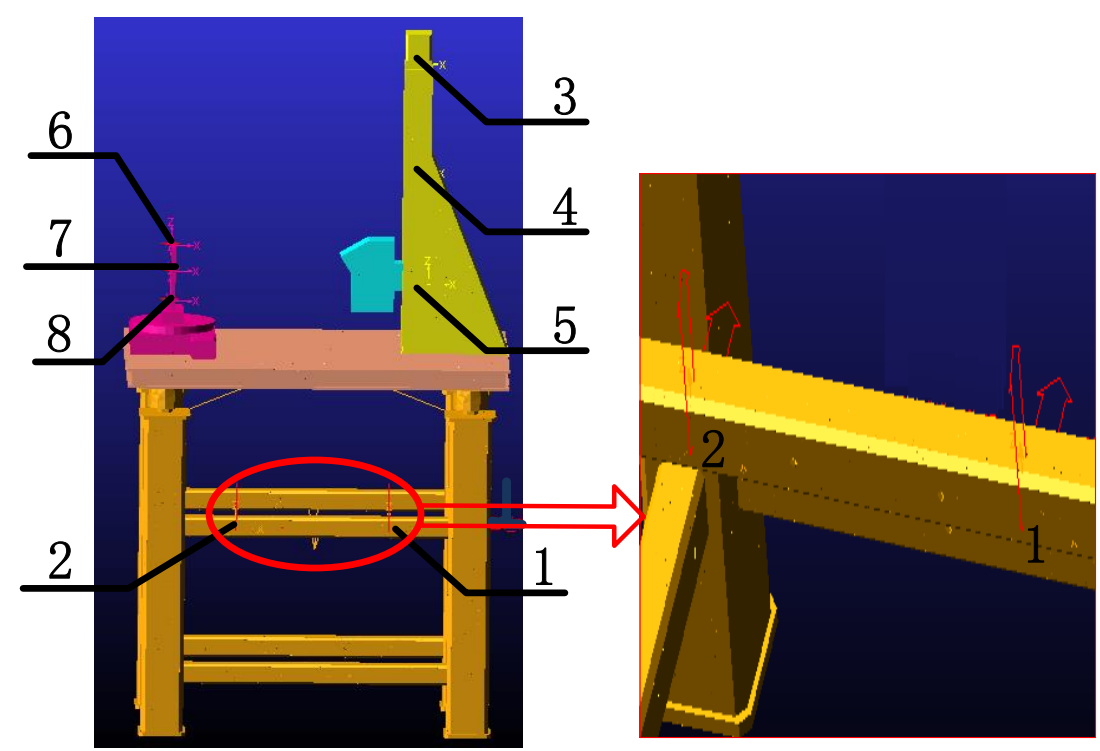

Figure 4. Inputs and outputs on the device

Simulation of horizontal vibration. The horizontal vibration of the input 1 was added to the device, and the responses of the output 3 8 are shown in Fig. 5. 


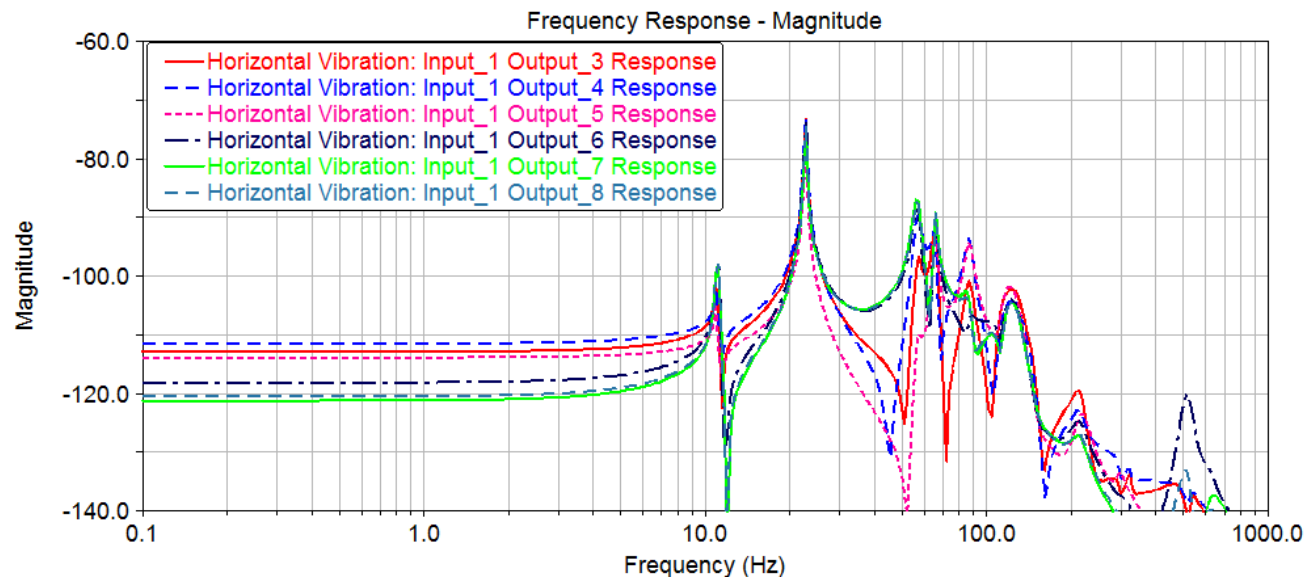

Figure 5. The horizontal vibration of the input 1

The horizontal vibration of the input 2 was added to the device, and the responses of the output $3 \sim 8$ are shown in Fig. 6.

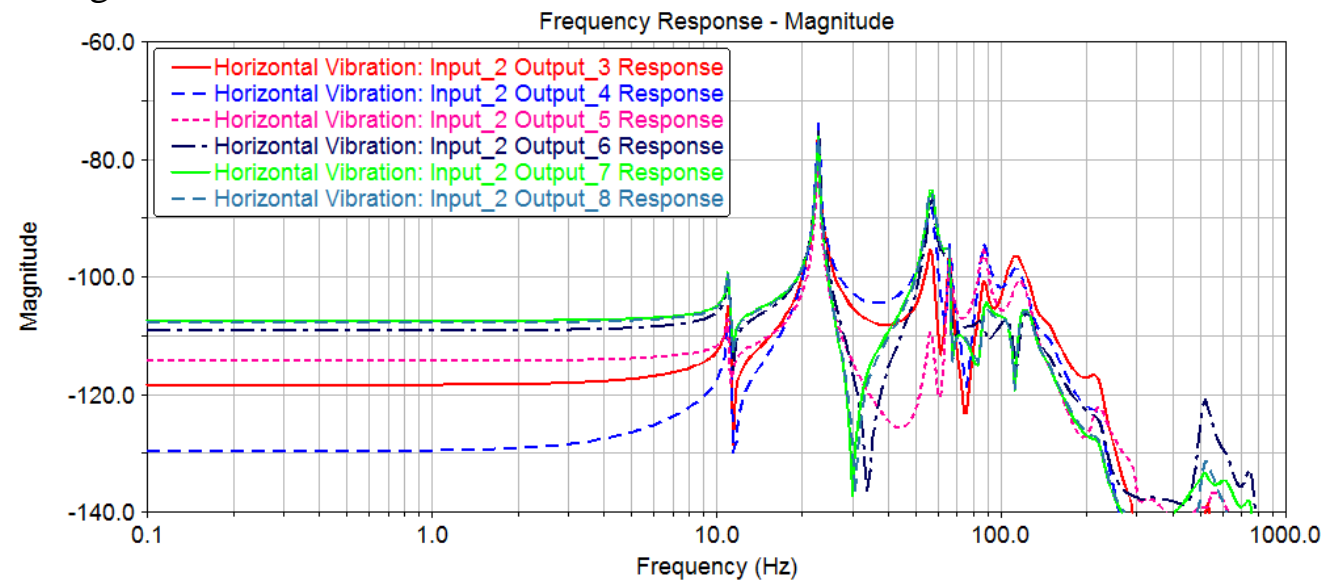

Figure 6. The horizontal vibration of the input 2

Simulation of vertical vibration. The vertical vibration of the input 1 was added to the device, and the responses of the output 3 8 are shown in Fig. 7 .

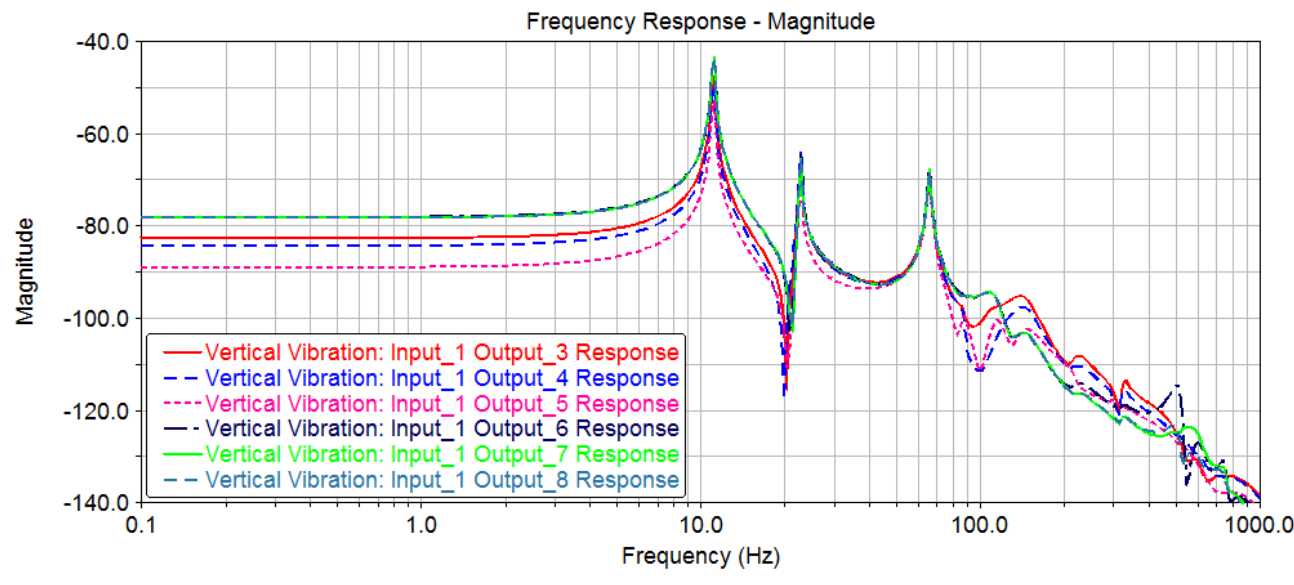

Figure 7. The vertical vibration of the input 1

The vertical vibration of the input 2 was added to the device, and the responses of the output $3 \sim 8$ are shown in Fig. 8. 


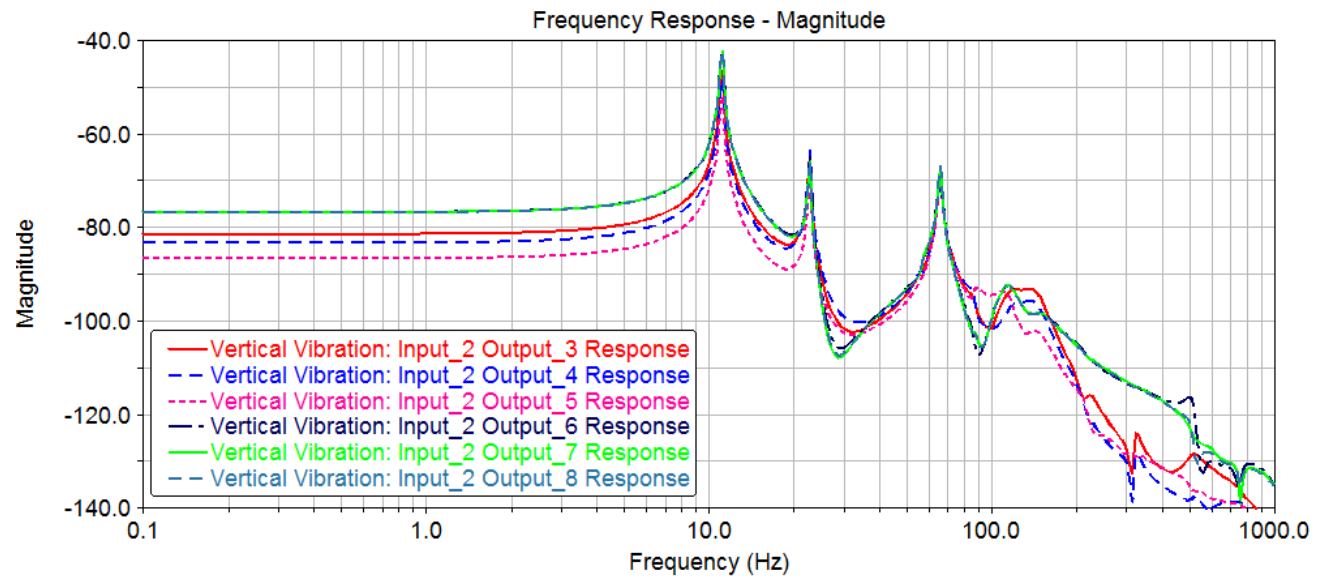

Figure 8 . The vertical vibration of the input 2

The variation trends of the vibration are similar at the six output points. The fluctuation range of the low frequency area $(0 \mathrm{~Hz} 10 \mathrm{~Hz})$ is very small. The energy is more concentrated and the fluctuations are more obvious between $10 \mathrm{~Hz}$ and $150 \mathrm{~Hz}$, and the amplitude of the frequency response has a sudden change at the intrinsic frequency.

Combining the above results with the environmental vibration value, it is concluded that there is a big influence on the precision of the device between $10 \mathrm{~Hz}$ and $150 \mathrm{~Hz}$. Thus, it is necessary to test the precision of the device under the environmental vibration by experiments.

\section{Experiments}

The vibration source is used to simulate the actual working condition of the device. Vibration test platform mainly consists of the following equipment: Modal 110 vibration exciter and MB 500 VI power amplifier produced by Dynamic MB Company, SCADAS III signal collection system produced by LMS Company. The measurement error of the device can be tested under the vibration, so we can determine whether the device meets the accuracy requirements in the factory environment. In this paper, the effects of vertical and horizontal vibrations on the device accuracy are considered.

4.1 Effect of horizontal vibration. Measuring of the standard ball diameter can determine whether the accuracy of the device meets the requirement. In the experiments the standard ball is shown in Fig. 9 and its diameter is $50.8055 \mathrm{~mm}$. When there is no external vibration source, the measured diameter of the standard ball is $50.7856 \mathrm{~mm}$. The horizontal vibration of the device is shown in Fig. 10, and the error of the device is measured by the random signals with different frequencies.

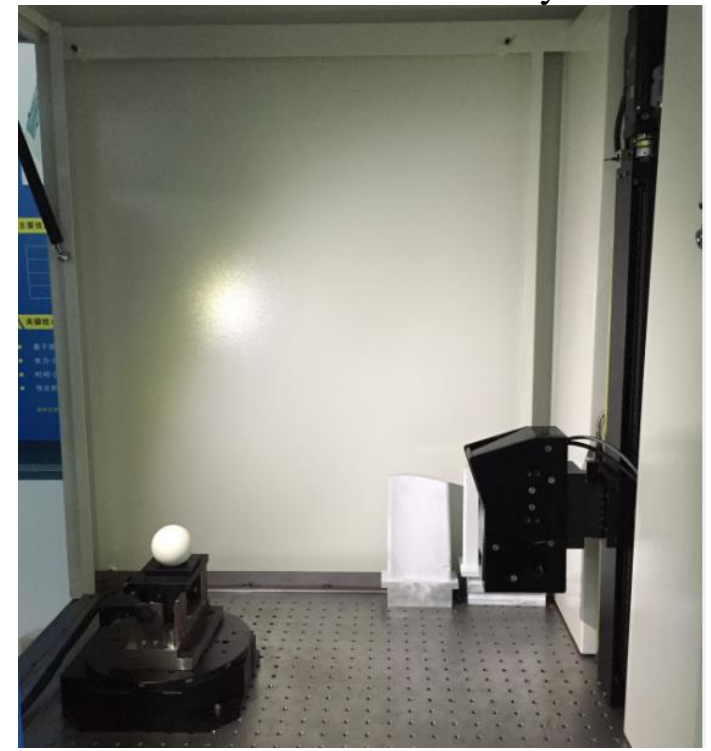

Figure 9. The measurement of the standard ball diameter

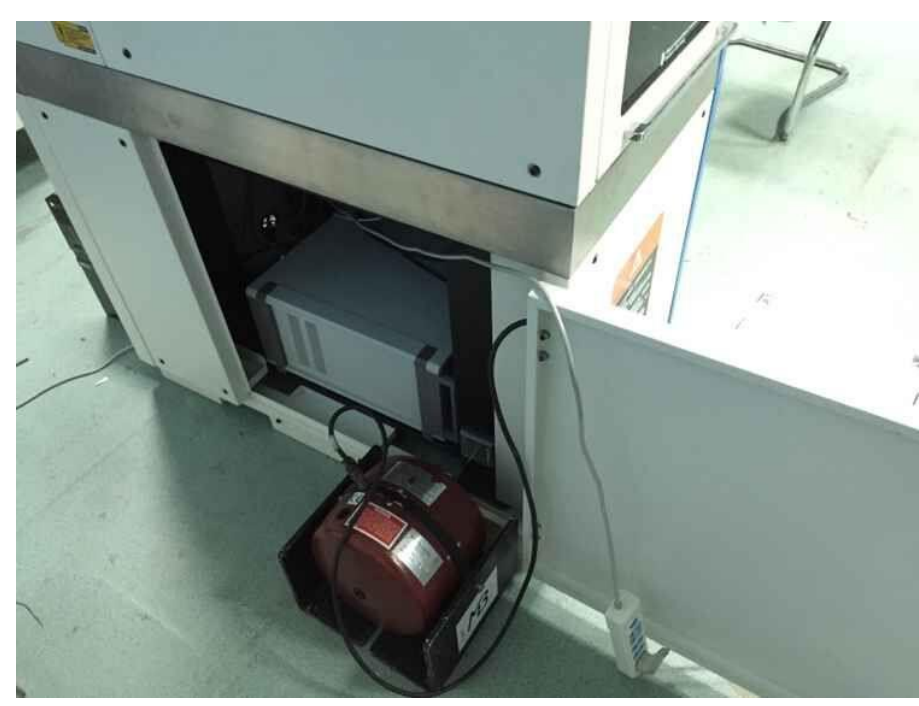

Figure 10. The horizontal vibration on the device 
When there is a horizontal vibration, the experimental results are shown in Table 4.

Table 4. The experimental results of horizontal vibration

\begin{tabular}{|c|c|c|}
\hline Frequency $(\mathrm{Hz})$ & Inspection diameter $(\mathrm{mm})$ & Absolute error $(\mathrm{mm})$ \\
\hline Without vibration & 50.7856 & -0.0199 \\
\hline $0 \sim 30$ & 50.7779 & -0.0276 \\
\hline $30 \sim 60$ & 50.7778 & -0.0277 \\
\hline $60 \sim 90$ & 50.7806 & -0.0249 \\
\hline $90 \sim 120$ & 50.7782 & -0.0273 \\
\hline $120 \sim 150$ & 50.7859 & -0.0196 \\
\hline
\end{tabular}

4.2 Effect of vertical vibration. The vertical vibration of the device is shown in Fig. 11. They represent that the vibration source is close to rotating stage and translation stage, respectively.

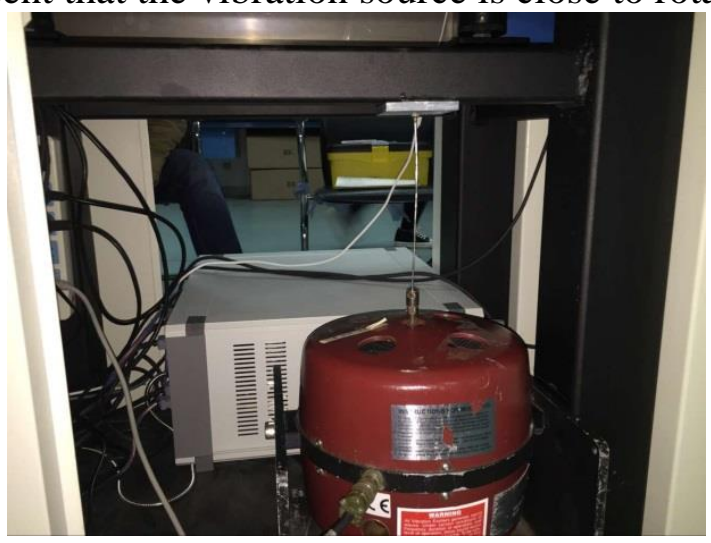

(a)



(b)

Figure 11. The vertical vibration on the device, (a) close to rotating stage and (b) close to translation stage

When there is a vertical vibration, the experimental results are shown in Table 5.

Table 5. The experimental results of vertical vibration

\begin{tabular}{|c|c|c|c|c|}
\hline \multirow{2}{*}{ Frequency $(\mathrm{Hz})$} & \multicolumn{2}{|c|}{ Close to rotating stage } & \multicolumn{2}{c|}{ Close to translation stage } \\
\cline { 2 - 5 } & Diameter $(\mathrm{mm})$ & Error $(\mathrm{mm})$ & Diameter $(\mathrm{mm})$ & Error $(\mathrm{mm})$ \\
\hline Without vibration & 50.7856 & -0.0199 & 50.7856 & -0.0199 \\
\hline $0 \sim 30$ & 50.7867 & -0.0188 & 50.7865 & -0.0190 \\
\hline $30 \sim 60$ & 50.7868 & -0.0187 & 50.7859 & -0.0196 \\
\hline $60 \sim 90$ & 50.7900 & -0.0155 & 50.7808 & -0.0247 \\
\hline $90 \sim 120$ & 50.7789 & -0.0266 & 50.7877 & -0.0178 \\
\hline $120 \sim 150$ & 50.7901 & -0.0154 & 50.7873 & -0.0182 \\
\hline
\end{tabular}

\section{Conclusions}

The vibration sensitive frequency range for precision device is $1 \mathrm{~Hz} \sim 150 \mathrm{~Hz}$ by relevant standards. This article figures out and testifies that the former 6 intrinsic frequency of the device is $19.68 \mathrm{~Hz}$, $24.23 \mathrm{~Hz}, 33.49 \mathrm{~Hz}, 49.64 \mathrm{~Hz}, 55.4 \mathrm{~Hz}$ and $63.2 \mathrm{~Hz}$, respectively. When the frequency of external vibration is between $10 \mathrm{~Hz}$ and $150 \mathrm{~Hz}$, there is a big influence on the precision of the device. At last, it is concluded that the error of the device is smaller than the allowable error under environmental vibration. The precision of the blade inspection can reach $0.0277 \mathrm{~mm}$, which meets the requirement of 
blade inspection device very well. The inspection device can be gradually applied into the margin detection and deformation correction of nuclear power forged blades.

\section{Acknowledgment}

This work is supported by the National Natural Science Foundation of China (No. 51475187), the National Basic Research Program of China (No. 2015CB057304), the Guangdong Innovative Research Team Program (Grant No. 2011G006) and the Open Funding of State Key Laboratory of Digital Manufacturing Equipment and Technology (Grant No. DMETKF2015002).

\section{References}

[1] W.L. Li, L.P. Zhou and S.J. Yan. 2015. A case study of blade inspection based on optical scanning method, International Journal of Production Research, 53(7): 2165-2178.

[2] T.H. Hsu, J.Y. Lai and W.D. Ueng. 2006. On the development of airfoil section inspection and analysis technique, The International Journal of Advanced Manufacturing Technology. 30(1): 129-140.

[3] T. Chen, X.M. Du, M. Jia and G. Song. 2010. Notice of Retraction Application of optical inspection and metrology in quality control for aircraft components, 2010 2nd International Conference on. IEEE, 5: V5-294-V5-298..

[4] E.Y. Heo, D.W. Kim, J.Y. Lee and K.Y. Kim. 2008. Computer-aided measurement plan for an impeller on a coordinate measurement machine with a rotating and tilting probe, Robotics and Computer-Integrated Manufacturing, 24(6): 788-795.

[5] Y.Q. Li, J. Ni. 2009. Constraints based nonrigid registration for 2D blade profile reconstruction in reverse engineering, ASME Journal of Computing and Information Science in Engineering, 9(3): 031005.

[6] R.A. Amy, G.S. Aglietti and G. Richardson. 2009. Reliability analysis of electronic equipment subjected to shock and vibration-a review, Shock and Vibration, 16(1): 45-59.

[7] X.B. Duan, Z.Y. Hao. 2009. Simulation of Engine Block Vibration for an Automotive Diesel Engine, I. C. E \& Powerplant, 26(4): 16-19.

[8] E. Celebi, G. Schmid. 2005. Investigation of ground vibrations induced by moving loads, Engineering Structures, 27(14): 1981-1988.

[9] L.J. Zhao, T. Xu and J.Liu. 2006. Modeling and Analysis on Vertical Vibration of Mill Using ADAMS/Vibration, Journal of System Simulation, 18(6): 1566-1569.

[10] Y.R. He. 2013. Response of Ultra-precision Machine under Micro-environment Vibration, Master thesis: Nanjing University of Science \& Technology. 